\title{
CONSIDERACIONES TEÓRICAS DE ESTRATEGIA Y GESTIÓN EN EL CONTEXTO DE LAS COOPERATIVAS
}

\author{
THEORETICAL CONSIDERATIONS STRATEGY AND MANAGEMENT IN THE \\ CONTEXT OF THE COOPERATIVE
}

\author{
Madelin Sánchez Otero** \\ Luisa Sánchez otero*** \\ Corporación Universitaria de la Costa CUC
}

RECIBIDO: Septiembre 3 de 2013

ACEPTADO: Noviembre 11 de 2013

\section{Resumen}

El presente artículo es de revisión bibliográfica, cuyo objetivo fue analizar referentes teóricos y empíricos sobre estrategia y gestión en las cooperativas. Se consultó fuentes secundarias como: artículos, libros físicos y electrónicos, bases de datos e informes y estadísticas institucionales. Se halló que existen pocos estudios sobre estrategia en el ámbito cooperativo y respecto al tema de gestión, la mayoría de estudios encontrados son empíricos. Se concluye, que la estrategia es una poderosa herramienta que facilita la gestión de directivos de una organización y la gestión está relacionada con la capacidad de los directivos para

\footnotetext{
** Administradora de Empresas, Magister en Dirección Estratégica, Investigadora del Grupo de Investigación Administración Social de la Corporación Universidad de la Costa, CUC. Correo: msanchez6@cuc.edu.co

*** Investigadora colaboradora, Administradora de Empresas, presidente de La Asociación Organización Popular de Vivienda Servicios Integrales a la Comunidad. Correo: luisa2504@hotmail.com
} 
ejecutar la estrategia; en las cooperativas, la estrategia y gestión representa un proceso de renovación más abierto y articulado con los retos del entorno.

Palabras claves Estrategia, organización cooperativa, gestión, participación, asociados. 


\begin{abstract}
This item is from literature review aimed at analyzing theoretical and empirical references on strategy and management in cooperatives. Secondary sources were consulted as articles, physical and electronic books, databases and reports and institutional statistics. It was found that there are few studies on the cooperative strategy and field management on the issue, most empirical studies are found. We conclude that the strategy is a powerful tool that makes managing directors of an organization and management is related to the ability of managers to execute strategy, in cooperatives, strategy and management represents a renewal process more open and articulated the challenges of the environment.
\end{abstract}

Key words: Strategy, cooperative organization, Management, Participation

Associates. 


\section{INTRODUCCIÓN}

El estudio de la estrategia y gestión ha sido un campo de estudio amplio, el cual ha contado con notables aportes de disciplinas como: sociología, economía, administración de empresas, entre otras. Los principales aportes han sido focalizados en el sector privado, especialmente para las empresas con ánimo de lucro. Sin embargo, en las últimas décadas, por la importancia y relevancia que se le ha dado al tercer sector a nivel mundial; a partir de la promulgación de los Objetivos del Desarrollo del Milenio (ODM) y el pacto mundial, el cual integra todas las organizaciones sin ánimo de lucro, ha sido de interés para autores e investigadores cuya línea de trabajo son temas como: administración, estrategia, gestión, competitividad y sostenibilidad.
En razón de ello, el tema de estrategia y gestión en el marco de las organizaciones cooperativas es importante estudiarlo, en tanto contribuye a identificar y establecer aspectos de la dinámica de estas organizaciones que tienen un aporte significativo a la economía del país y se crean como respuesta a las necesidades de un grupo de personas que desean de manera colectiva solucionarlas.

El sector cooperativo en Colombia, según la Confederación de cooperativas de Colombia ${ }_{1}$ Confecoop (2011) es representativo para el desarrollo del país, esto se ve reflejado en su aporte al PIB de un $4,7 \%$. Sin embargo, las cooperativas micro, pequeñas $y$ medianas presentan deficiencias en términos de debilidad en su estructuración, organización y 
economía. Así mismo, limitaciones

en términos de competitividad.

El objetivo del artículo es establecer marco de referencia teórico-conceptual sobre la estrategia y gestión en el ámbito de las organizaciones cooperativas, a partir de la revisión de teóricos y estudios empíricos.

Inicialmente se aborda, la conceptualización de lo que es una organización cooperativa, según la institucionalidad, seguidamente aspectos generales relacionados con la finalidad del artículo. Posteriormente, se hace revisión de respecto a la gestión y estrategia en las organizaciones cooperativas, para obtener la información se revisaron investigaciones orientadas a estudiar, caracterizar o proponer lineamientos del desempeño general de estas organizaciones.
En relación a la estrategia, se

establecen concepciones y posiciones de reconocidos autores en el tema de estrategia. De igual forma, se revisan artículos y estudios focalizados sobre el tema de estrategia concretamente en las organizaciones cooperativas. En relación a esto último, se encontró muy poca evidencia. Los estudios o investigaciones están orientados a temas más amplios como la competitividad y el desempeño general.

La organización

\section{cooperativa}

Para la Alianza Cooperativa Internacional $(\mathrm{ACl})$, una cooperativa es:

"una asociación autónoma de personas que se han unido de forma voluntaria para satisfacer sus necesidades $\quad y \quad$ aspiraciones económicas, sociales y culturales en 
común mediante una empresa de propiedad conjunta y de gestión democrática ( $\mathrm{ACl}, 2009)$._Es decir, surge a partir de la iniciativa de un grupo de emprendedores de una zona específica (Coque, 1999)

En Colombia, La Ley 79 de 1988 define que la cooperativa es una empresa asociativa sin ánimo de lucro, en la cual los trabajadores o los usuarios, según el caso, son simultáneamente los aportantes y los gestores de la empresa, con el objetivo de producir, distribuir conjunta y eficientemente bienes o servicios para satisfacer las necesidades de sus asociados y de la comunidad en general.

Las anteriores definiciones de cooperativa desde un ámbito institucional, tienen elementos comunes como son: empresas sin ánimo de lucro, se crean voluntariamente, lo emprendedores son aportantes y gestores de la empresa. Estas similitudes hacen de la empresa cooperativa, una organización particular, porque su fin no es económico, sino social. Sin embargo, las organizaciones cooperativas necesariamente tienen que lograr sinergia entro lo económico y social, para perdurar en el tiempo. La visión empresarial no puede ser ajena a ellas; como lo afirma Rivera et al (2009) el fracaso sería inmediato al estar alejado de la práctica contemporánea empresarial.

Entre otras características propias del modelo cooperativo, se destaca: el tema de propiedad es totalmente diferente en comparación con las empresas lucrativas.

\section{Gestión cooperativa}


Desde la academia y el sector cooperativo se realizan

Investigaciones desde diversas perspectivas y disciplinas, entre ellas: la dirección y gestión. A fin de contextualizar lo anterior, está la investigación realizada por Bastidas (1998), quien afirma que la focalización que tienen las organizaciones en lo social no ha permitido que estas empresas tengan una visión empresarial y por tanto quienes dirigen están maniatados para actuar. Según Davis (1999), las organizaciones cooperativas no tienen la conciencia o compromiso de establecer buenas prácticas administrativas y ni dan importancia a liderazgo que deben ejercer para lograr mantener los principios cooperativos.

Otro estudio es el de Carroz (2005) denominado: "Modelo de gestión estratégica para el desarrollo de capacidades tecnológicas".

Identificaron analfabetismo de los sisaleros y la falta de competencia para sostenerse un mercado agresivo. A través de esta investigación logró incrementar la competitividad del sector sisalero.

En un entorno cambiante, las empresas cooperativas tienen necesidad de responder oportunamente a todos los cambios, logrando sinergia entre los principales actores (asociados, empleados, directivos y comunidad); quienes contribuyen a lograr resultados superiores, en la medida que tienen bien definido su papel o rol en el desarrollo de la organización. También, debe ser clara la labor de la asamblea de socios, la cual define los lineamientos estratégicos para la organización y vela por los intereses de la cooperativa y sus asociados, Es la asamblea conjuntamente con 
la dirección de la cooperativa; quienes se encargan de maximizar los beneficios, producir ingresos para la cooperativa, generar bienestar a sus empleados y a los asociados especialmente. Es decir, concentrar sus esfuerzos

principal mente en que los asociados participen en las asambleas y en la capacitación, pues es allí donde se refleja la gobernabilidad de estas organizaciones (Salazar, 2010.Pág. 129). Aspecto importante que plantea Senge (2005), cuando afirma que tener una visión genuina permite que la gente haga lo que le corresponde, sin que se lo impongan.

Sin embargo, en las organizaciones cooperativas el cuadro directivo tiene mucho más poder, que todos sus miembros; esto obedece generalmente a la débil influencia que ejercen los mismos y por tanto el cuerpo directivo estará menos presionado para dar resultados y mantener cercanía con los asociados (Spear, 2004). En el mismo sentido, Puentes (2008) afirma que los asociados generalmente no hacen uso de sus derechos y deberes, además no asisten a los espacios que tienen para tomar decisiones como son las asamblea, dejándole así el poder al consejo.

De acuerdo al párrafo anterior y según Hernández et al (2006, pág. 3), en estudio realizado a más de 40 organizaciones se identificó que presentaban dificultades en: el control, el manejo de las crisis, el manejo de estrategias, el manejo de procesos, el desarrollo de nuevas ideas, la investigación, resultan con bajísimos niveles de desarrollo, lo 
cual denota baja capacidad

operativa de tales empresas.

Teniendo en cuenta lo descrito en párrafos anteriores, otro aspecto fundamental para el desarrollo de las cooperativas es el aprovechamiento de los avances tecnológicos en todos los procesos de la organización. Sin duda, esto facilita y mejora la prestación de servicios, los procesos productivos y de gestión,

Esto se fundamenta en lo expuesto por Breen (2008,a): la gestión basada en el control y la eficiencia no son suficientes para el entorno actual.

El Instituto para la promoción y la formación Cooperativa realizó un estudio dirigido a 92 cooperativas exitosas, en éste se demostró que es mayor la probabilidad de éxito de una cooperativa cuando colabora con otra (Amat, 1997).
Desde su experiencia, Hamel (2007) considera que la mayoría de las personas orientan su atención en el cumplimiento y la eficiencia. Sin embargo, para crear una capacidad de innovación constante y rápida, es necesario depositar la responsabilidad por la innovación avanzada en los dueños de los procesos internos. Hamel hace énfasis en que el crecimiento y sostenimiento de las compañías es responsabilidad de quienes trabajan en ellas, desde luego con un líder a la cabeza.

Así lo define Breen (2008,b, pág.14): las empresas deben poder ser tan estratégicamente adaptables como ya son operativamente eficientes. Lo descrito hasta el momento, orienta a las cooperativas a generar cambios en su forma de gestionar y esto se logra definitivamente siendo estratégicos desde la dirección de las empresas, 
logrando la contribución de todos los miembros de la compañía y realizar alianzas estratégicas para construir redes que le permita fortalecer procesos y servicios. Sin embargo, Kornitz (2011) plantea que construir organizaciones $y$ redes más amplias, esto a menudo resulta contradictorio con la dinámica propia de sus actividades cotidianas y la dedicación que requieren para el sostenimiento mismo de la unidad.

Lo anterior coincide con los resultados de la investigación realizada por Bastidas \& Enciso (2006), quienes encontraron que las organizaciones solidarias tienen dificultad para establecer interlocución con otras organizaciones, situación que incide ante el Estado, la empresa privada, los medios de comunicación y la comunidad, para influir en políticas públicas, articular recursos y generar sinergias, lo que merma su reconocimiento y posicionamiento. Al respecto, Cuñat y Coll (2008, pag 25) afirman que: la gestión de la cooperativa es uno de los temas olvidados por éstas. Los socios suelen estar preocupados por el trabajo diario. Lo cual tiene incidencia en las condiciones de la organización, porque actúan de acuerdo a las situaciones que se presenten.

\section{Estrategia}

El término estrategia ha sido usado durante siglos y ha ido evolucionando en el tiempo, sobre todo en el campo de los negocios, el uso de este término se restringía al campo militar y al mundo de la diplomacia.

Se le atribuye a Drucker (1980), la utilización del término 
estrategia en el campo de la administración, para el autor, el concepto aplicado a la organización, responde a dos preguntas: ¿Qué es nuestro negocio? y ¿Qué debería ser? Estas preguntas invita a las compañías a reconocer su posición actual a fin de de que pudieran definir su futuro teniendo claro en que negocios querían estar.

Otro de los autores líderes en este campo, es Chandler (1962). Para él la estrategia es la determinación de metas y objetivos básicos de largo plazo de la empresa, la adición de los cursos de acción y la asignación de recursos necesarios para lograr dichas metas. Según el autor la estructura organizacional sigue la estrategia; en este sentido, su interés estaba puesto en estudiar la relación entre la forma que las empresas seguían en su crecimiento (sus estrategias) y el diseño de la organización (su estructura) planeado para poder ser administrada en su crecimiento.

Kenneth Andrews combina las ideas de Drucker y Chandler. En este sentido, define la estrategia como un conjunto de decisiones que revela las metas y las principales políticas o planes para alcanzarlas. Así mismo, define en el negocio que está o debiese estar la empresa, el tipo de organización además de precisar la naturaleza de la contribución económica y no económica que busca realizar a sus accionistas, empleados, clientes y a la comunidad.

Ansoff (1998) desarrolló su concepto de estrategia "desde el interior" de la empresa. En este sentido, sus tres preocupaciones principales fueron la agresividad 
estratégica (el nivel de

discontinuidad que la empresa introduce en la generación de sus productos y tecnología), la respuesta de la capacidad de la empresa (que debe acoplarse a la turbulencia del entorno) y la adopción de decisiones estratégicas (centrándose en cómo y cuándo deben ser adoptadas para maximizar la rentabilidad económica, no la contable, de la empresa).

Para Porter (1996), estrategia es construir una posición única y valiosa en el mercado, sobre la base de un conjunto de actividades específicas y únicas que posea una empresa. Para otros autores cómo Fred R (2003) las estrategias son acciones potenciales que requieren decisiones de parte de la gerencia y de recursos de la empresa. Igualmente Hitt, Irelan y
Hoskisson (2007, pág. 4) definen la estrategia como: un conjunto de compromisos y acciones, integrados y coordinados, diseñados para explotar las competencias centrales y lograr una ventaja competitiva.

Hill y Jones (2009, pág. 3) conciben la estrategia así: conjunto de acciones estructuradas que los administradores adaptan para mejorar el desempeño de su compañía. Los autores fundamentan este concepto en el hecho que para la mayoría de empresas el reto máximo es lograr un desempeño superior al de sus rivales. En consecuencia, si la estrategia de una compañía logra un desempeño superior, se dice que hay una ventaja competitiva. De allí la importancia de una buena dirección por parte del administrador; a fin de identificar las áreas problemáticas, elaborar 
alternativas y proyectar la acción, pero la dificultad del administrador no es la de la comprensión teórica de los conceptos de administración sino la aprehensión de su metodología de diseño e implementación (Zabala, 2005)

Considerando lo anterior, las organizaciones cooperativas pueden hacer de su naturaleza solidaria una ventaja competitiva. También, _Cárdenas y Dávila (2006) en su artículo titulado: ¿Puede el tercer sector construir capital social? plantean que "el sector solidario, por su parte, también ayuda en la construcción de capital social y se puede considerar como una herramienta de generación de equidad social. Para Izquierdo (2005, pág.8): estas iniciativas, además de presentar muchas de las cualidades de las PYME que se han puesto en marcha recientemente, ofrecen ventajas especiales derivadas de su forma cooperativa: a) Su independencia de la inversión exterior les permite generar empleo, allí donde las empresas tradicionales no lo hacen, b) sus bases de asociación pueden hacer de ellas empresas social, económica y tecnológicamente innovadoras, c) su estructura organizativa promueve el espíritu de empresa entre grupos, d) sus amplios objetivos impulsan el bienestar y la prosperidad de muy diversos interesado y e) sus estructuras participativas pueden materializar, con la cooperación entre esos diversos interesados, el potencial local o regional que se halle en estado latente.

De acuerdo, a lo plateado por los autores estudiados, la estrategia involucra: las acciones, es decir para está claro que la estrategia son 
las acciones que requiere una organización para lograr lo que desea. Así mismo, de manera Entre estos implícita o explícita está presente el tema de las decisiones y los objetivos o propósitos a alcanzar orientados a lograr un mejor desempeño.

En síntesis, la estrategia en términos administrativos básicamente se puede entender como la adaptación de los recursos y habilidades de la organización a un entorno que cambia constantemente (Porter, 1982), aprovechando las oportunidades presentes y evaluando los riesgos en función de objetivos y metas. Las prácticas gerenciales en las organizaciones apuntan a generar estrategias que permitan enfrentar crisis, contrarrestar una situación riesgosa o liderar el mercado a través de una mayor diferenciación, rentabilidad, permanencia y crecimiento.

\section{Estrategia en el contexto de} las organizaciones cooperativas

Diversos autores han desarrollado estudios orientados a caracterizar o mejorar la gestión y competitividad del sector cooperativo. En este sentido, los resultados contribuyen como herramientas o modelos de mejora para la gestión y competitividad de las empresas cooperativas.

De acuerdo a lo anterior, se cita la investigación realizada por Morales en el año 2002. En esta, se evidencio que se vislumbra un imaginario social integral, donde viabiliza los modelos que ponen en práctica la Teoría O, concebida esta como un modelo multicausal, donde se destaca la cultura organizacional, una administración favorable, condiciones externas que no 
hipotequen el desarrollo dada la importancia que tienen empresarial, entre otros aspectos y estos aspectos para el crecimiento y principalmente se premia la sostenibilidad de las empresas en el participación y la confianza entre los miembros. Este imaginario no se da por procesos formales, se establece a partir de la cultura que se genere desde la dirección, buscando eliminar las diferentes barreras internas y propiciando los enfoques multidisciplinarios e independientes. En síntesis, para que la organización cooperativa alcance buenos resultados se requiere institucionalizar la participación desde todos los ámbitos, sobre todo por la naturaleza y características propias de este tipo de empresas.

Según estudio realizado por Monteguth (2006), el cual tenía como propósito principal analizar los factores de éxito competitivo y las variables de gestión de las almazaras cooperativas catalanas, 
crédito. Por ello, el principal propósito fue mejorar desde la perspectiva financiera la compresión del papel clave de los activos intangibles en el valor real de una organización. Los principales resultados de esta investigación fueron: en cuanto al personal, se da estabilidad, por la política de reclutamiento, selección y promoción del personal que tiene la cooperativa. Además, la estabilidad, se relaciona directamente con la apropiación de los intangibles y desde luego con una menor fuga de conocimiento organizativo. Otra conclusión importante que arrojó el estudio de caso, es la política de desarrollo profesional e integración del personal, la cual se traduce en mejora de las aptitudes y el incremento de valor de los intangibles. Siguiendo con los resultados obtenidos en la investigación, se destacan otros como: el alto sentido de pertenencia, compromiso, auto motivación del personal; resultado asociado a la cultura de "clan", el clima laboral y los traslados internos que ha desarrollado la cooperativa. La conclusión final, respecto a las preguntas de investigación fue: que el tipo de empresa, es decir la forma jurídica, tiene incidencia en la conformación del personal, sobre todo en los intangibles relacionados con los valores y las actitudes. Todos estos resultado están relacionados con la fuerte cultura que ha desarrollado la empresa cooperativa.

Consecutivamente, se consultó a Dávila (2008), quien participó en una investigación, utilizando metodología de estudios de caso realizado entre el año 1993 y 2005. Los estudios de casos tenían como objetivo establecer, 
cómo las cooperativas hacen parte de un paradigma de ventaja competitiva, los fundamentos de ese paradigma, la especificidad cooperativa, la particularidad de la gestión y la organización cooperativa. Los resultados de investigación se integran a una investigación internacional: Internan Innovative Programme Investigación, que se viene realizando en Asia, África y Latinoamérica. Entre los principales resultados de esta investigación están: organizaciones privadas con preocupaciones públicas y privadas, organizaciones basadas en el poder de la acción colectiva, organizaciones fundamentadas en la cultura local, organizaciones y empresas diferentes, organizaciones que desarrollan una gestión social basada en valores, la cooperativa; una empresa de participación, el liderazgo; un elemento fundamental. En síntesis, se concluyó que el modelo organizacional y empresarial de las cooperativas por sus propias características y elementos permite construir ventajas competitivas.

En igual sentido, los investigadores Moyano, Puig y Bruque (2008) llevaron a cabo un estudio denominado "Los determinantes de la competitividad en las cooperativas", el objetivo principal de esta investigación fue: evaluar el papel que juegan otros elementos desde los que la literatura ha estudiado o aproximado con referencia a la competitividad como son: el entorno, características internas relacionadas con los procesos de producción y la comercialización. Según los resultados de esta investigación: son los factores internos que 
determinan la competitividad de las empresas.

Por otro lado, March, y Yagüe

(2009) realizan el estudio

"Desempeño de empresas de economía social. Un modelo para su

medición." Cuyo propósito fue:

Identificar si el colectivo de empresas de economía social tenían resultados "singulares", muy diferentes a los de las otras compañías. Los principales resultados de este estudio fueron: $\mathrm{E}$ perfil de las empresas estudiadas revela una rentabilidad menor a pesar del crecimiento que ha tenido en ventas en los últimos anos, por encima de las empresas convencionales, también revela un comportamiento dispar de una variable entre las empresas autogestoras. Se trata del indicador costos de ventas/ ventas por empleado.
En este contexto de consultas, esta De Melo y Pulido (2010). El objetivo principal de la investigación que realizaron fue: analizar la gestión de la compañía Cooptur en un momento específico, pero con el fin de identificar $y$ avanzar en el conocimiento de los factores que afectan a la gestión de cooperativas brasileñas en el ámbito turístico. Entre los principales resultados de esta investigación están: La importancia de la formación de los gestores de las cooperativas, la variable a la que mayor importancia le dan es la de aspectos internos, olvidándose de la variable externa; según el estudio porque son más complejos de manejar. Así mismo, la educación cooperativa no aparece con alto grado de importancia porque es una cooperativa reciente y no han recibido la capacitación. La gestión no es estructurada, es decir, 
solucionan los problemas del día a día y no se evidenció una El estudio realizado por planificación a largo plazo.

Pabón \& Romero (2010), sobre la Evaluación del sistema de gestión

Otro estudio, realizado por Sánchez, O (2010, pag.8,9), se encontró, que las principales dificultadas que presentan las cooperativas desde la dirección son: falta de un plan educativo en formación y capacitación (técnicaeducación cooperativa); existe desconocimiento y falta de participación en actividades que ofrecen entidades públicas y privadas para el desarrollo del sector (proyectos sociales, económicos); los asociados manifestaron que la principal causa de insatisfacción es "la faltad e información de los servicios y beneficios"; también, que en ocasiones los costos de estar en la cooperativa son más altos que los beneficios que reciben. de tres formas asociativas rurales en el municipio de Tabio (Cundinamarca), arrojo como resultado deficiencias en: desconocimiento de la política pública, débil compromiso de asociados y de la junta, no tienen capacitación en solidaridad y por tanto desconocen la importancia de la solidaridad.

En síntesis, quienes dirigen y representan las organizaciones cooperativas, alternativa que contribuye al desarrollo de las comunidades, tienen la responsabilidad de hacerlas eficientes. Para Bernal (2006) el éxito de las cooperativas radica en el compromiso con la comunidad o su entorno próximo 
Lo que implica, lograr

sinergia entre los diferentes actores, transparencia en sus acciones y sobre todo excelente gestión para lograr la longevidad de la organización (Puentes et al., 2009). Por tanto, la reflexión y la gestión estratégica es una tarea urgente en las empresas de economía solidaria (Sanchis \& Campo, 2007b). Para Izquierdo (2005) las cooperativas deben responder "al proceso internacional de competencia y concentración" para lograr competitividad; pero sin perder su naturaleza. Especialmente atender las demandas de sus asociados o propietarios. $\mathrm{Y}$ esto se logra a través de procesos estratégicos, que necesariamente debe involucrar a todos los actores de la organización cooperativa para la formulación (Malo, 2006).
En este mismo orden de ideas, Ramírez (2002) citado por Dávila R (2004, pág. 35-36), plantea que el paradigma de gestión estratégico como el conjunto de valores y supuestos de la gestión que le da legitimidad al despliegue de las acciones estratégicas de la organización en cuestión y establece tres aspectos claves que son el fundamento de la ventaja cooperativa.

a) El propósito de la formulación de la estrategia: está focalizado en el servicio y a la calidad de éste, permitiendo establecer diferencias de la gestión cooperativa claramente con la gestión en general. b) Filosofía de gestión, que se define como el conjunto de valores y supuestos en los que enmarca y se justifican las acciones cotidianas de la gestión estratégica, siendo de gran 
importancia la concordancia y articulación entre el contenido teórico de la estrategia y la ejecución del proceso.

c) El modo de gestión de la formulación estratégica de la cooperativa, asociado a dos aspectos básicos, la "concepción" de la estrategia y el de "liderazgo" en el proceso, los cuales tienen injerencia directa en el estilo de gestión y hacen parte de la una de las características de la estrategia cooperativa.

Desde esta perspectiva, según Hernández, P (2001), las empresas cooperativas necesitan implementar un sistema estratégico para mejorar su sistema de gestión.

\section{Hallazgos}

A partir del análisis precedente de la revisión de los diferentes teóricos y de estudios empíricos se establecieron los principales hallazgos así:

De la búsqueda y revisión de la literatura, se encontró que existen muy pocos estudios focalizados en el tema de estrategia en el sector cooperativo. Respecto al tema de gestión, la mayoría de las investigaciones encontradas son estudios empíricos.

Las organizaciones cooperativas tienen una singularidad y es la propiedad y la participación de sus miembros. De la revisión de las diferentes investigaciones o estudios también se revela la incidencia que tienen los individuos en los resultados, especialmente sus asociados. Pese a lo anterior, Morales, A. C. (2004) afirma que los asociados tienen cierto nivel de desconfianza con los consejos de administración porque 
no están satisfechos con la

información que les entregan y sienten estar distantes de la dirección. Ahora bien, la importancia que la literatura le da a los asociados en los resultado de la empresas cooperativa, está asociado a la capacidad y habilidad que tengan los directivos para ver qué es un "activo estratégico complejo y único, conformando una fuente significativa de heterogenidad y de ventaja competitiva sostenible para las organizaciones" (Pastor, 2011, pag. 148).

Es posible establecer, que existen muchas herramientas y factores que coadyuvan al éxito de una organización, pero sin duda alguna es el enfoque claro y la toma de decisiones asertivas que aseguran mejores resultados y llevan a una empresa desarrollar ventajas competitivas.
Actualmente el sector cooperativo tiene dificultades en términos de la identidad cooperativa y de los valores. Se dan dos aspectos relacionados con lo anterior: el poco sentido de pertenencia de los asociados y una dirección no tan democrática, centrada solo en las operaciones internas de la empresa.

\section{Las} organizaciones necesariamente tienen que conciliar lo económico con lo social; estas no pueden medir tanto sus resultados en términos cuantitativos, porque el éxito de éstas, está realmente en lo social. No quiere decir esto, que las cooperativas no deben preocuparse por la parte cuantitativa, desde luego que es importante y es uno de un factor para mantenerse en el largo plazo. La naturaleza misma de estas empresas, debe ser un 
orientador para quienes las dirigen, por ejemplo: Procurar buenas prácticas de gestión de la mano con la responsabilidad social.

El gran desafío de los directivos 0 de quienes dirigen organizaciones de naturaleza cooperativa es lograr la participación de todo su grupo de interés en los resultados. Este cambio debe ser administrado y planeado tal como lo plantea Ventosa y Villegas (2009): Aquí es donde descansa la principal labor de los dirigentes del sector cooperativo, en ayudar a que las organizaciones desarrollen estructuras y procesos que favorezcan el progreso pleno de las personas involucradas, de sus funcionarios y asociados.

Hay diferencias entre las cooperativas grandes y pequeña y micro (clasificación según
Confecoop), estas diferencias están marcadas en términos financieros, de gestión y resultados. Muchas cooperativas grandes tienen muy buenos resultados económicos pero se ha diluido el tema de propiedad e identidad cooperativa. En lo que respecta a las cooperativas micro, adicionalmente al tema de propiedad e identidad cooperativa, se le suma la falta de capital, el poco nivel de formación de todos, precaria gestión, orientadas a la parte interna y generalmente tienen un solo cliente.

Hoy, cualquier empresa para ser sostenible en el largo plazo, necesariamente tiene que tener estrategas, porque el tema de apertura de fronteras ha generado grandes cambios a nivel de negocios y estos cambios deben ser administrados. Así sea una empresa sin ánimo de lucro, tiene que generar excedentes para lograr 
cumplir su razón de ser. La cual es: contribuir al desarrollo del ser humano. En tal sentido, Sanchis \& Campos( 2007a) plantea que el crecimiento de este tipo de organizaciones está asociado a la innovación social; en tanto es posible ratificar un posicionamiento estratégico que le permite desarrollar ventajas competitivas.

En su gran mayoría, los autores consultados que han realizado investigaciones sobre organizaciones cooperativas, plantean cambios significativos en la dirección de las empresas cooperativas, reafirmando que el éxito de la organización cooperativa radica en la inclusión de los asociados en la gestión. Es decir, se privilegia la participación, adicionalmente se requiere de propuestas innovadoras en los emprendimientos sociales. Las corrientes de pensamiento se enmarcan en aspectos como: las características propias del modelo cooperativo, tal como lo propone Eguía (2009, pág.7): Esbozar a una cooperativa que favorezca el desarrollo económico y social de sus componentes $y$ de sus comunidades.

Es evidente, que en términos de estrategia y gestión a nivel de cooperativas micro, pequeña y mediana se requiere mayor acompañamiento del Estado en las acciones que se emprenden, buscando fortalecer el sector desde la formulación y ejecución de la política pública. También, es importante que las agremiaciones tengan mayor incidencia en la formulación de políticas públicas para el sector de la economía solidaria y desde luego sean estratégicas para impulsar redes de cooperación entre las empresas del 
sector; para lograr impacto en la calidad de vida de las personas que le apuestan a la creación de estas organizaciones, con el sueño de lograr satisfacer necesidades que individualmente no pueden alcanzar. Se debe apuntar a la búsqueda de acuerdos entre los diferentes actores del mercado (Malo, 2003). En igual sentido, García, G (1998) y Cegarra, et al (2005), plantean que las organizaciones, para conseguir una adecuada competitividad necesariamente tienen trabajar en red. Según, Lundvall (2002) el hecho de establecer redes contribuye a la generación de conocimiento e innovación; siendo este uno de los elementos claves de éxito de algunas cooperativas estudiadas. Al respecto, Colina et al. (2006) indican que los procesos de innovación solo se dan si las personas que integran la organización tienen las capacidades para generar estos.

\section{Conclusiones}

Los estudios de estrategia y gestión cooperativa han sido muy poco estudiados en Colombia, aun cuando es un sector que aporta a la economía del país y sus organizaciones presentan dificultades y limitaciones recurrentes.

Se infiere la importancia del tema de estrategia y gestión en el ámbito de cualquier organización para su crecimiento y competitividad. Por lo que, su interpretación, adecuación, aplicación y control representa un reto para los directivos de toda organización.

El tema de gestión y estrategia en el marco de las organizaciones cooperativas es una 
necesidad apremiante; para ello, se requiere de estrategas con un enfoque social en la dirección, a fin de lograr sinergia entre lo económico y los principios cooperativos.

En el ámbito de las organizaciones cooperativas, los asociados tienen un papel fundamental, siempre y cuando los directivos los involucren en la concepción y desarrollo de la estrategia y la gestión.

Finalmente, el uso de la estrategia y gestión en las organizaciones cooperativas requiere cambios de paradigmas de quienes dirigen; desarrollo de habilidades gerenciales tanto de directivos como de asociados; impulsar la autogestión y el empoderamiento de los asociados para lograr resultados sostenidos y multiplicadores. Desde el Estado se requiere un real acompañamiento para generar competencias en los individuos y no dependencia, contextualizado a las condiciones de estas iniciativas colectivas que se dan de manera voluntaria 0 impulsada por programas o proyectos privados o públicos, sobre todo para las cooperativas micro y pequeña.

\section{Referencias}

$\mathrm{ACl} \quad-\quad$ ALIANZA COOPERATIVA INTERNACIONAL (2009). Ley marco para las cooperativas dEe América Latinala ed. -- San José, Costa Rica: Alianza Cooperativa Internacional para las Américas. Recuperado Marzo 13 de 2012.

http://www.aciamericas.coop/IMG/p df/LeyMarcoAL.pdf 
Amat

O. (1997). Estudio

Bernal,

A. (2006),

"La

socioeconómico de las cooperativas

a Catalunya, Institut per a la

promoción i la formacio

Cooperatives, Barcelona, 1997.

Ansoff Igor (1998), "The New

Corporate Strategy". Publicado por

Wiley and Sons (NY), 227- 235.

Prentice Hall International (Uk).

Bastidas, O (1998). Apuntes para el conocimiento del movimiento cooperativo venezolano. CIRIECEspaña, 1998, nº 30, pp. 81-101

Bastidas, A, Luis \& Enciso, J. Carlos (2006) Promoción, desarrollo y fortalecimiento del sector de economía solidaria en el departamento de Nariño. Recuperado mayo de 2011 http://www.revistamemorias.com/edi cionesAnteriores/8/promociondesarr ollofortalecimientoeconomiasolidaria pasto.pdf globalizacióny el

cooperativismo", en Cuadernos

Cooperativosy de

Economía Social, núm. 14,

México.

Breen, H. (2008). El futuro de la administración.

Bogotá:

Editorial Norma S.A.

Cárdenas y Dávila (2006) Derecho a Solidarizarse. Compilado por DANSOCIAL. Segunda versión. Bogotá: Editorial: Imprenta Nacional de Colombia.

Carroz D. (2005). Modelo de gestión estratégica para el desarrollo de las capacidades tecnológicas, Revista Compendium. Venezuela.

Chandler A. (1962). "Strategy and Structure": Chapters in the history of American Industrial Enterprise, The MIT Press. 
Cegarra, Juan, Briones, Antonio \& Ros, María del Mar (2005) La confianza como elemento esencial para la mejora de la cooperación entre empresas: un estudio empírico en pymes. Cuadernos de Administración Vol. 18 No. 30, Pág.

22. Bogotá Junio a Diciembre

Confecoop (2011). Desempeño cooperativo Colombiano 2011. http://www.confecoop.coop/images/i nformes_anuales/2011.pdf Julio 31 de 2012

Colina, B, Petit, E \& Gutiérrez, I (2006) Gestión de conocimiento para liberar el poder de la innovación como fuente de ventajas competitivas en las organizaciones, Espacios, 27, 2006, p. 26-38.

Colombia (1998) Ley 79 Congreso de la Republica de Colombia. Diario oficial 36.648. Bogotá DC.

Coque, J (199). Industrialización en el entorno local a través de (sociedades) cooperativas. Revista de Estudios Cooperativos (REVESCO), 1999, No 68, pp.119137

Cuñat, R \& Coll, V (2008) Nuevas cooperativas de trabajo asociado: Un análisis del proceso de creación basado en la Teoría Fundamentada. Revista Venezolana de Economía Social Año 8, No 15, Enero-Junio. Universidad de los Andes (ULA)

Dávila R (2004) Innovación y éxito en la gerencia cooperativa casos exitosos de cooperativas rurales de ahorro y crédito. Fundación Cultural Javeriana de Artes

Gráficas - JAVEGRAF. Bogotá.

Bogotá, D.C., 2004Dávila R. (2008). Cooperación y desarrollo local: cooperativas rurales de ahorro $y$ crédito del Sur de Santander, Colombia: UniRcoop Vol. 6, \# 1, 2008. 
Davis, P. (1999). La administración de la Diferencia Cooperativa. Desarrollo del recurso humano para la administración y el establecimiento de redes de cooperativas, conjuntamente con la División Cooperativa de la Oficina Internacional del Trabajo. Ginebra. Ginebre: Edición de COOPNET -

De Melo M. Pulido J (2010) La gestión cooperativa del turismo en Brasil. El caso de la cooperativa Paranaense de Turismo. Ciriec España, Revista de Economía Publica, Social y Cooperativa, Volumen, no. 67, Abril 2010, pp.243-268.

Drucker, P. (1980). Managing in Turbulent Times. Ed. Harpers, N.Y.

Eguía, f. (2009) la cooperativa para el desarrollo económico y socialdocumento de discusión. I Cumbre Cooperativas de las Américas.
México. Recuperado agosto 2012. http://www.aciamericas.coop/cumbr e2009/IMG/pdf/Florencio Eguia-

Cumbre Mexico eje3.pdf

Fred, D. (2003). Conceptos de administración estratégica. México, Pearson Educación.

García, G (1998) El micro emprendimiento y las empresas de participación. Revista Revesco, No 65

Hamel, G. (2007). El futuro de la Administración. Harward Business School Press. Grupo. Ciudad: Bogotá. Editorial Norma.

Hernández F. (2001). La Economía Cooperativa Como Alternativa Empresarial. España: Universidad de Castilla- La Mancha.

Hernández, V, Albeiro, Lasso, Norma, Cardona, Gloria, Mosquera, John \& Londoño, Alexandra (2006) 
Evolución y estado actual del sector de la economía solidaria en los municipios de Pereira, Dosquebradas y Santa Rosa de Cabal. (Risaralda). Recuperado mayo de 2011 http://www.revistamemorias.com/edi cionesAnteriores/8/evolucionestado actualeconomisolidaria.pdf
Hill,
C. y Jones
G. 2009 .

Administración Estratégica. México: Octava edición. Mc Graw Hill.

Hitt, Irelan y Hoskisson (2007). Administración estratégica, competitividad $y$ globalización. México: $7^{a}$ edición, Cengage Learning,

Izquierdo, C. (2005) El cooperativismo una alternativa de desarrollo a la globalización neoliberal para América Latina, Edición electrónica a texto completo en

www.eumed.net/libros/2005/ceia/.

Kenneth Andrews (1971). "Andrews, K. (1971). En: The Concept of Corporate Strategy. Homewood III. Dow- Jones Irwin., Preface III

Kornitz (2011) "Estrategias de sostenibilidad en la Economía del Trabajo: los casos de Pashuca y Tinta Roja". La revista del CCC [en línea]. Septiembre / Diciembre n13. Recuperado junio 10 del 2013.http://www.centrocultural.coop/ revista/articulo/298/. ISSN 18513263.

Lundvall, B. (2002). EstadosNación, capital social y desarrollo económico. Un enfoque sistémico de la creación de conocimiento y el aprendizaje en la economía global; en Revista de Economía Mundial no $7,69-90$ 
Malo, M.C. (2003) "La Cooperación

y la Economía Social". M. VUOTTO (comp.)

Economía Social Precisiones

Conceptuales y Algunas

Experiencias Históricas. Buenos

Aires: Ed. Altamira

Malo, Marie Claire. (2006). Cooperativas y modelo de desarrollo: la experiencia quebequense. Cayapa. Revista Venezolana de Economía Social, Sin mes, 12-29. Recuperado abril 20 de 2012 http://www.redalyc.org/articulo.oa?id $=62261103$

March, I. Yagüe, R. (2009) Desempeño de empresas de economía social. Un modelo para su medición. . Ciriec - España, Revista de Economía Publica, Social y Cooperativa, Vol. 64, Abril 2009, pp.105-131.
Monteguth, Y. (2006) Investigación titulada Análisis de los factores de éxito competitivo de las Almazaras cooperativas Catalanas.

d'Administració d'Empreses i Gestión Económica de los Recursos Naturals Facultad de Dret i Economía Universidad de Lleida. Recuperado agosto de 2012 http://www.tdx.cat/bitstream/handle/ 10803/8065/Tyms1de1.pdf;jsessioni $d=C 5 F 547 E F 097464 A 14 C B 34 C 48 A$ 05AFBCA.tdx2?sequence $=1$

Morales, F. (2002) El imaginario social de la participación y el modelo cooperativo como arquetipo empresarial tipo "O". CIRIECEspaña, Revista de Economía Pública, Social y Cooperativa, Volumen No. 40, Abril 2002, pp. 4371.

Morales, A. C. (2004). La dirección en la empresa de trabajo asociado: 
una revisión de estudios Pastor, S. Enrique (2011) empíricos. CIRIEC-España, Revista Participación ciudadana, desarrollo de Economía Pública, Social y e intervención comunitaria, capital Cooperativa,(48) 99-122. social, calidad democrática, cambio Recuperado de social. Revesco No 104 - Primer http://redalyc.org/articulo.oa?id=174 Cuatrimestre 2011. Recuperado 04806 $\begin{array}{lll}\text { abril de } & 2012\end{array}$ Moyano, J., Puig F. Bruque S. (2008) Los determinantes de la competitividad en las cooperativas" CIRIEC-España, Revista de Economía Pública, Social y Porter, M,E (¡982) Estrategia Cooperativa, Núm. 61, pp. 233-249. Competitiva: Técnicas para el Pabón, P, \& Romero, F (2010), Evaluación del sistema de gestión de tres formas asociativas rurales en el

http://pendientedemigracion.ucm.es/ info/revesco/txt/REVESCO\%20N\%2 0104.6\%20Enrique\%20PASTOR\%2 OSELLER.pdf análisis de los sectores industriales y de la competencia. Edición I. Compañía Editorial S.A. de C.V. (México).

municipio de Tabio

(Cundinamarca)", en Revista Porter, M. E (1996) "What Is Cooperativismo y Strategy?" Harvard Business Desarrollo, vol. 18, núm. 97, Review, 74, no. 6 (Novemberpp. 90-104. December).

Puentes, R.: (2008) "Las cooperativas de segundo grado 
como forma de integración: especial referencia al efecto impositivo", Edición electrónica gratuita. Texto completo

en

www.eumed.net/tesis/2008/rpp/

Puentes, R.; Velasco,M \& Vilar,J (2009) $\therefore$ El Buen Gobierno Corporativo en las Sociedades Cooperativas. REVESCO: Revista de Estudios Cooperativos, $\mathrm{n}^{\circ}$. 98, Sin Mes, 2009 , p. $118-140$

Rivera, R,C, Alfonso, $\mathrm{A}, \mathrm{J}$ \& Labrador, M.O (2009) retos y perspectivas del cooperativismo, como real alternativa de desarrollo ante la globalización neoliberal. Revista avances, Ciget Pinar del rio, Vol. 11 No. 1 enero. Recuperado en $\begin{array}{lll}\text { abril } & \text { de }\end{array}$ http://www.ciget.pinar.cu/Revista/No .2009

1/articulos/Retos_perspectivas_Coo perativismo..pdf
Salazar, L. (2010),

“¿Cómo funciona el gobierno cooperativo en las cooperativas de Villavicencio?", en Revista Cooperativismo y Desarrollo, vol. 18, núm. 97, pp. 126-138.

Sánchez M (2010). SITUACIÓN ACTUAL Y PERSPECTIVAS DE LAS COOPERATIVAS MICRO. Económicas CUC/Volumen 31, Número 31/Enero - Diciembre de 2010/Barranquilla - Colombia/ISSN 01203932.

Sanchis, Juan \& Campo, Vannesa (2007) La innovación social en la empresa, el caso de las cooperativas y de las empresas de economía social en España. Revista Economía Industrial No 368. Recuperado agosto 15 de 2012 de http://www.mityc.es/Publicaciones/P ublicacionesperiodicas/Economialnd 
ustrial/RevistaEconomialndustrial/36

8/187.pdf

Seguí, E. (2007) Tesis doctoral "La

gestión del capital intelectual en las

entidades

financieras.

Caracterización del capital humano en las cooperativas de crédito.

España: Universidad Politécnica de Valencia. Recuperado abril 20 de 2012

de

http://riunet.upv.es/bitstream/handle/ 10251/1836/tesisUPV2605.pdf

Senge, P. (2005) La quinta disciplina: el arte y la práctica de la organización abierta al aprendizaje, Buenos Aires $2^{\mathrm{a}}$ ed, $4^{\mathrm{a}}$ reimp: Centro de Publicaciones Educativas y Material Didáctico. : Granica.

Spear, R. (2004). El gobierno democrático en las organizaciones cooperativas. CIRIEC-España, Revista de Economía Pública, Social y Cooperativa, (48) 11-30.
Recuperado

de

http://www.redalyc.org/articulo.oa?id

$=17404802$

Ventosa, D. Villegas, G. (2009) Las

cooperativas como parte de la economía social ¿una alternativa real ante la crisis?: una aproximación desde el desarrollo organizacional.-documento de discusión-

http://www.larepublica.com.co/archiv os/PYMES/2010-07-29/ingresos-delas- cooperativas-representan-5-delpib_106705.php.

Zabala, H. (2005). Planeación estratégica aplicada a cooperativas y demás formas asociativas y solidarias. Bogotá. Editorial: Universidad Cooperativa de Colombia. 